\title{
Protective effects of dantrolene and methylprednisolone against spinal cord injury-induced early oxidative damage in rabbit bladder: A comparative experimental study
}

\author{
Ibrahim Keles ${ }^{1, A, B, D}$, Mehmet Fatih Bozkurt ${ }^{2, A, D}$, Erdogan Aglamis ${ }^{3, C, D}$, Abdurrahman Fatih Fidan ${ }^{4, C, F}$, \\ Cavit Ceylan ${ }^{5, D, E}$, Mustafa Karalar ${ }^{1, D, F}$, Soner Coban ${ }^{6, B, C}$, Baris Denk $^{4, B, C}$, Mehmet Emin Buyukokuroglu ${ }^{7, E, F}$ \\ ${ }^{1}$ Department of Urology, Faculty of Medicine, Afyon Kocatepe University, Afyonkarahisar, Turkey \\ 2 Department of Pathology, Faculty of Veterinary, Afyon Kocatepe University, Afyonkarahisar, Turkey \\ ${ }^{3}$ Clinics of Urology, Elazig Training and Research Hospital, Saglik Bilimleri University, Turkey \\ ${ }^{4}$ Department of Biochemistry, Faculty of Veterinary, Afyon Kocatepe University, Afyonkarahisar, Turkey \\ ${ }^{5}$ Clinics of Urology, Turkey Yuksek Ihtisas Training and Research Hospital, Saglik Bilimleri University, Ankara, Turkey \\ ${ }^{6}$ Clinics of Urology, Sevket Yilmaz Training and Research Hospital, Saglik Bilimleri University, Bursa, Turkey \\ ${ }^{7}$ Department of Pharmacology, School of Medicine, Sakarya University, Turkey \\ A - research concept and design; $\mathrm{B}$ - collection and/or assembly of data; $\mathrm{C}$ - data analysis and interpretation; \\ $D$ - writing the article; $E$ - critical revision of the article; $F$ - final approval of the article
}

Address for correspondence

Ibrahim Keles

E-mail: drkeles@hotmail.com

\section{Funding sources}

This study was supported by the Afyon Kocatepe University Scientific Research Projects Coordination Unit (Project No. 14.TIP.09).

Conflict of interest

None declared

Received on March 8, 2018

Reviewed on 0ctober 8, 2018

Accepted on June 27, 2019

Published online on December 18, 2019

Cite as

Keles I, Bozkurt MF, Aglamis E, et al. Protective effects of dantrolene and methylprednisolone against spinal cord injury-induced early oxidative damage in rabbit bladder: A comparative experimental study. Adv Clin Exp Med. 2019;28(12):1697-1704. doi:10.17219/acem/110326

DOI

10.17219/acem/110326

Copyright

Copyright by Author(s)

This is an article distributed under the terms of the

Creative Commons Attribution 3.0 Unported (CC BY 3.0)

(https://creativecommons.org/licenses/by/3.0/)

\section{Abstract}

Background. Spinal cord injury (SCI) may cause dysfunction in the bladder and many distal organs due to systemic inflammatory response and oxidative stress-related injury.

Objectives. We investigated the preventive effects of dantrolene (DNT) and methylprednisolone (MP) on stress-induced tissue damage in rabbit bladder with SCl.

Material and methods. A total of 35 rabbits were included in this study and they were divided into 5 groups: group 1 - control, group 2 - SCl only, group 3 - SCl and DNT, group 4 - SCl and MP, and group 5 - SCI and DNT+MP. Twenty-four hours after SCl, the bladders of these rabbits were removed and the histopathologic changes in the bladder were examined under a light microscope. Additionally, malondialdehyde (MDA), glutathione (GSH), and nitric oxide (NO) levels were evaluated as antioxidant agents both in bladder tissue and in blood.

Results. Compared to the control group, there was an increase in edema and congestion in all groups. The least amount of edema was observed in the group receiving DNT and the least amount of congestion was observed in the group receiving combined treatment (group 5). No superiority was found between the drug-receiving groups in terms of reducing MDA level in blood and tissue after SCl. The most successful group was the group receiving combined drug therapy in terms of increasing the blood GSH level, which was significantly decreased after SCl. After SCl, blood NO level increased significantly in all groups. Nitric oxide levels in the bladder tissue significantly decreased in the groups receiving DNT and combination therapy and fell in the control group.

Conclusions. Dantrolene and MP may have potential benefits against oxidative damage in the bladder after SCIs because of their anti-inflammatory and antioxidant effects. In particular, the combined use of DNT and MP at different doses can be considered a treatment strategy.

Key words: antioxidant, anti-inflammatory, spinal cord injury, methylprednisolone, dantrolene 


\section{Introduction}

Based on several regional studies, the annual incidence of spinal cord injury (SCI) in the USA is estimated to be roughly 12,000 new cases per year. ${ }^{1}$ Spinal cord injuries are mostly caused by traumatic incidents such as traffic accidents, firearm injuries, falls and sports injuries, but nontraumatic causes such as intraspinal infection, vascular ischemia and tumor may also cause SCIs. ${ }^{2}$ Traumatic SCI can cause serious neurological damage and multiple organ dysfunction in patients. ${ }^{2,3}$ Traumatic SCI is a 2-phase pathological process defined as primary injury and secondary injury. ${ }^{2,4}$ Physical compression and mechanical injury of the spinal cord after trauma is defined as primary injury. ${ }^{4,5}$ Primary injury causes spinal deformation and narrowing of the spinal canal, resulting in intraspinal hemorrhage and decreased blood circulation due to mechanical damage of nerve tissue and blood vessels. ${ }^{2}$ Primary injury occurs in a short period of time and in a restricted area. Hemorrhage is a process characterized by ischemia and edema. ${ }^{2}$ Primary injury triggers secondary injury that may occur within hours or days. ${ }^{6}$

Major pathophysiological changes observed in the secondary injury phase include: reduction in glutathione levels, ischemia, oxidative damage, $\mathrm{Ca}^{2+}$-dependent nitric oxide (NO) production, excitotoxicity, free radical damage, lipid peroxidation in cell membranes, increased malondialdehyde level, which is the end product of membrane lipid peroxidation, neurodegeneration, gliosis, and inflammation. ${ }^{2,7-9}$ The purpose of medical treatment of traumatic SCIs is to prevent the effects of these secondary mechanisms. ${ }^{10}$ In addition to intraspinal inflammation, SCI can trigger systemic inflammatory response syndrome (SIRS), which can lead to failure and dysfunction in multiple organs. ${ }^{2}$ After SCI, in the urinary system, complications such as neurogenic bladder, kidney damage and urinary tract infection can be observed. ${ }^{2}$ Loss of neuronal stimulation and inflammation play a role in the pathogenesis of urinary system dysfunction. ${ }^{2}$

The inhibition of inflammatory responses can contribute to the recovery from neurogenic depression. ${ }^{2}$ Antioxidant and anti-inflammatory agents such as dantrolene (DNT) and methylprednisolone (MP) are used for this purpose. ${ }^{11}$ Dantrolene is a ryanodine receptor antagonist that blocks the intracellular release of $\mathrm{Ca}^{2+}$, and is used as an anti-inflammatory and neuroprotective agent. ${ }^{11}$ Methylprednisolone acts as a steroidal anti-inflammatory agent, reducing the number of inflammatory cells and oligodentrocytic apoptosis. ${ }^{11}$

The purpose of our work is to comparatively demonstrate the protective effect of DNT and MP, individually or in combination, on stress tissue damage in rabbit bladder in experimental SCI.

\section{Material and methods}

The study was conducted in accordance with the Guide for the Care and Use of Laboratory Animals published by the US National Institutes of Health (NIH Publication No. 85-23, revised 1996) and was approved by the Afyon Kocatepe University Animal Experiment Ethics Committee, Afyonkarahisar, Turkey (approval No. AKUHADYEK-49533702/59).

\section{Animals}

A total of $35 \mathrm{New}$ Zealand male rabbits weighing $2.5-3 \mathrm{~kg}$ were included in the study. The animals were kept in individual cages, under a circadian cycle and temperature control in addition to standard feeding, at the laboratory animals center.

\section{Drugs and chemicals}

The drugs and chemicals used were the following: DNT sodium (Ryanodex vial 250 mg/20 mL, Eagle Pharmaceuticals, Woodcliff Lake, USA), MP sodium succinate (Prednol-L amp. $250 \mathrm{mg} / 4 \mathrm{~mL}$, Mustafa Nevzat Pharmaceuticals, Istanbul, Turkey), ketamine hydrochloride (Ketalar $50 \mathrm{mg} / \mathrm{mL}$; Pfizer, New York, USA) $25 \mathrm{mg} / \mathrm{kg}$, and xylazine (Rompun 100 mg/mL, Bayer AG, Leverkusen, Germany) $5 \mathrm{mg} / \mathrm{kg}$ injection. All drugs were diluted in $0.9 \%$ sterile saline.

\section{Experimental design and administration of drugs}

The animals were randomly divided into 5 groups consisting of 7 rabbits each, as follows: group 1 (control group) rabbits did not receive any drugs and no operation was applied; in group 2 rabbits (SCI+no drug treatment group) a single dose of $2 \mathrm{~mL}$ saline was given intraperitoneally (i.p.) $1 \mathrm{~h}$ after SCI; group 3 rabbits (SCI+DNT group) were administered $10 \mathrm{mg} / \mathrm{kg}$ DNT i.p. $1 \mathrm{~h}$ after SCI; group 4 rabbits (SCI+MP group) were given $30 \mathrm{mg} / \mathrm{kg}$ MP i.p. $1 \mathrm{~h}$ after $\mathrm{SCI}$; and group 5 rabbits (SCI+DNT+MP group) were administered 10 mg/kg DNT+30 mg/kg MP $1 \mathrm{~h}$ after SCI.

\section{Surgical procedures for the $\mathrm{SCl}$ model}

An appropriate disinfection and sterilization environment was established. All rabbits were anesthetized with intramuscular (i.m.) ketamine hydrochloride $25 \mathrm{mg} / \mathrm{kg}$ and xylazine $5 \mathrm{mg} / \mathrm{kg}$ injection. If necessary, the dose was repeated at $25 \mathrm{mg} / \mathrm{kg}$ ketamine and $5 \mathrm{mg} / \mathrm{kg}$ xylazine. During ketamine anesthesia, spontaneous breathing of the animals was observed. After back-shaving and field treatment, the rabbits were placed in prone position on the operation table. A midline dorsal incision was performed. The laminae and transverse processes of T6-L2 were exposed with gentle blunt dissection of the paravertebral muscles. 
A self-retaining retractor was placed in the operation area, and then laminectomy was performed at T10; then a balloon angioplasty catheter (Medtronic-146.671, 2.0×20 mm; Medtronic plc, Dublin, Ireland) placed extradurally and sublaminary on thorasic spinal cord, upwards below T9 and inflated under $2 \mathrm{~atm}$ pressure for $5 \mathrm{~min}$ with standby. Following the careful removal of the balloon catheter, the paravertebral fascia and skin were sutured with nonabsorbable sutures. Paraparesia developed in all groups, constituting traumatic injury. Twenty-four hours after SCI, the rabbits in all groups were anesthetized with $25 \mathrm{mg} / \mathrm{kg}$ ketamine and $5 \mathrm{mg} / \mathrm{kg}$ xylazine. The bladder was removed after an abdominal incision. Blood samples from each group were collected with cardiac puncture into heparinized and non-heparinized tubes under anesthesia at the end of the study protocol. At the end of these procedures, all rabbits were sacrificed under deep anesthesia.

\section{Biochemical analysis}

Malondialdehyde (MDA), glutathione (GSH) and NO levels were assessed as antioxidant agents in both bladder tissue and blood in all experimental groups.

\section{Blood sample collection}

Two milliliters of blood were immediately pipetted into a separate tube to measure MDA and GSH. The remaining blood was centrifuged at 3,000 rpm for $10 \mathrm{~min}$ for plasma separation. Plasma samples were stored at $-30^{\circ} \mathrm{C}$ for the analysis of NO.

\section{Tissue homogenate}

At the end of the study protocol, the urinary bladder tissues were washed immediately with ice-cold $0.9 \% \mathrm{NaCl}$. The urinary bladder was trimmed free of extraneous tissue and rinsed in chilled $0.15 \mathrm{M}$ Tris- $\mathrm{HCl}$ buffer ( $\mathrm{pH}$ 7.4). These tissues were blotted dry and homogenized in $0.15 \mathrm{M}$ Tris$\mathrm{HCl}$ buffer (pH 7.4) to yield a 10\% (w/v) homogenate. Then, they were centrifuged at $2,100 \mathrm{~g}$ for $10 \mathrm{~min}$ at $4^{\circ} \mathrm{C}$. The pellets represented the nuclear fraction and the supernatants were subjected to centrifugation at 18,600 g for $20 \mathrm{~min}$ at $4^{\circ} \mathrm{C}$. Reactive oxygen species (ROS) generation was observed in all the fractions as well as the whole homogenate.

\section{Measurement of malondialdehyde, reduced glutathione and nitric oxide in blood and tissue homogenates}

Malondialdehyde levels, as an index of lipid peroxidation, were assayed using the thiobarbituric acid test as described by Ohkawa et al. ${ }^{12}$ The principle of the method is based on spectrophotometric measurement of the color produced during the reaction of thiobarbituric acid with MDA and its absorbance was measured spectrophotometrically at $532 \mathrm{~nm}$. Reduced GSH concentration was measured using the method described by Beutler et al. in whole blood and tissue homogenates. ${ }^{13}$ The optical density was measured at $412 \mathrm{~nm}$ in the spectrophotometer. Results were expressed as $\mathrm{nmol} / \mathrm{mL}$ in blood and $\mathrm{nmol} / \mathrm{g}$ in tissue. Plasma NO levels were analyzed with the vanadium (III) chloride Griess reaction method of Miranda et al. that can simultaneously determine the nitrite and nitrate levels in the sample. ${ }^{14,15}$

\section{Histologic analysis}

The histopathological changes were assessed with light microscopy by the same pathologist in the bladders removed $24 \mathrm{~h}$ after SCI. Urinary bladders were collected and divided into 2 equal parts. One of them was stored at $-20^{\circ} \mathrm{C}$ until biochemical examination. The other part was fixed in buffered $10 \%$ formalin solution for pathological examinations. After routine processing, the tissues were embedded in paraffin, sectioned and stained with hematoxylin \& eosin (H\&E). Stained sections were blindly analyzed under a light microscope (Olympus CX41 attached with Kameram ${ }^{\circledR}$ Digital Image Analyze System; Olympus, Tokyo, Japan) for inflammation, edema and congestion, and scored from 0 to 6 . The absence of these findings was evaluated as 0 , and the presence as mild to severe (1-6).

\section{Statistical analysis}

Statistical tests were performed using SPSS for Windows v. 20.0 software package (SPSS IBM, Armonk, USA). Variables were investigated using visual (histogram and probability plots) and analytical methods (KolmogorovSmirnov test) to determine whether or not they were normally distributed. The results are reported as mean \pm standard deviation (SD) or as median (min-max). Data with normal distribution was analyzed using one-way analysis of variance (ANOVA) and Tukey's post hoc test. We used statistical evaluation with a nonparametric Kruskal-Wallis test for data with abnormal distribution. The MannWhitney $U$ test was performed to analyze the 2 groups. P-value $<0.05$ was assessed as statistically significant.

\section{Results}

\section{Histological observations}

Within the first $24 \mathrm{~h}$ in terms of inflammation, no difference was observed between all groups $(p=0.3)$. This may be because inflammation is not fully developed in the first $24 \mathrm{~h}$ (Table 1). When edema was assessed, there was a significant increase in edema in all groups compared to the control group $(\mathrm{p}<0.001)$. There was no difference between groups 4 and 5 , whereas edema was less pronounced in the group receiving DNT $10 \mathrm{mg}$ alone. The edemadissolving property is higher in the DNT-treated group. 
Table 1. Histopathological and biochemical values of blood and tissue for MDA, GSH and NO in experimental groups (mean \pm SD)

\begin{tabular}{|l|c|c|c|c|c|c|}
\hline \multicolumn{1}{|c|}{ Examination } & Parameter & Control & SCl & SCI+DNT & SCI+MP & SCl+DNT+MP \\
\hline \multirow{3}{*}{ Pathology } & inflammation & $0.14 \pm 0.37$ & $0.14 \pm 0.37$ & $0.29 \pm 0.75$ & $0.29 \pm 0.48$ & $0.71 \pm 0.75$ \\
& edema & $0.0 \pm 0.0$ & $3.29 \pm 0.75^{\mathrm{c}}$ & $1.71 \pm 0.75^{\mathrm{b}}$ & $2.43 \pm 1.13^{\mathrm{c}}$ & $2.43 \pm 0.53^{\mathrm{c}}$ \\
\cline { 2 - 8 } & congestion & $0.71 \pm 0.95$ & $4.0 \pm 0.0^{\mathrm{c}}$ & $4.14 \pm 0.37^{\mathrm{c}}$ & $4.0 \pm 1.15^{\mathrm{c}}$ & $3.43 \pm 0.53^{\mathrm{c}}$ \\
\hline \multirow{3}{*}{$\begin{array}{l}\text { Blood } \\
\text { biochemistry }\end{array}$} & $\mathrm{MDA}[\mathrm{nmol} / \mathrm{mL}]$ & $3.88 \pm 0.29$ & $8.86 \pm 1.81^{\mathrm{b}}$ & $4.08 \pm 0.49$ & $4.59 \pm 0.84$ & $4.32 \pm 0.58$ \\
& $\mathrm{GSH}[\mathrm{mg} / \mathrm{dL}]$ & $15.11 \pm 1.56$ & $10.7 \pm 0.75^{\mathrm{b}}$ & $11.15 \pm 0.42^{\mathrm{b}}$ & $11.33 \pm 0.62^{\mathrm{b}}$ & $12.92 \pm 0.91^{\mathrm{a}}$ \\
\hline \multirow{2}{*}{$\begin{array}{l}\text { Tissue } \\
\text { biochemistry } \\
\text { (urinary bladder) }\end{array}$} & $\mathrm{NO}[\mu \mathrm{mol} / \mathrm{L}]$ & $10.01 \pm 1.79$ & $13.03 \pm 0.87^{\mathrm{a}}$ & $25.11 \pm 4.01^{\mathrm{b}}$ & $28.68 \pm 2.49^{\mathrm{b}}$ & $25.26 \pm 2.84^{\mathrm{b}}$ \\
\hline & $\mathrm{MDA}[\mathrm{nmol} / \mathrm{mg}]$ & $1.97 \pm 0.43$ & $3.98 \pm 1.82^{\mathrm{a}}$ & $1.63 \pm 0.45$ & $1.67 \pm 0.58$ & $1.57 \pm 0.26$ \\
\hline
\end{tabular}

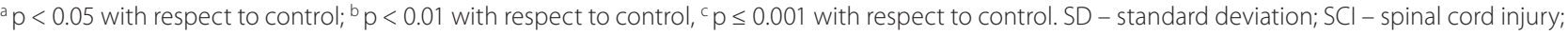
DNT - dantrolene; MP - methylprednisolone; MDA - malondialdehyde; GSH - glutathione; NO - nitric oxide.

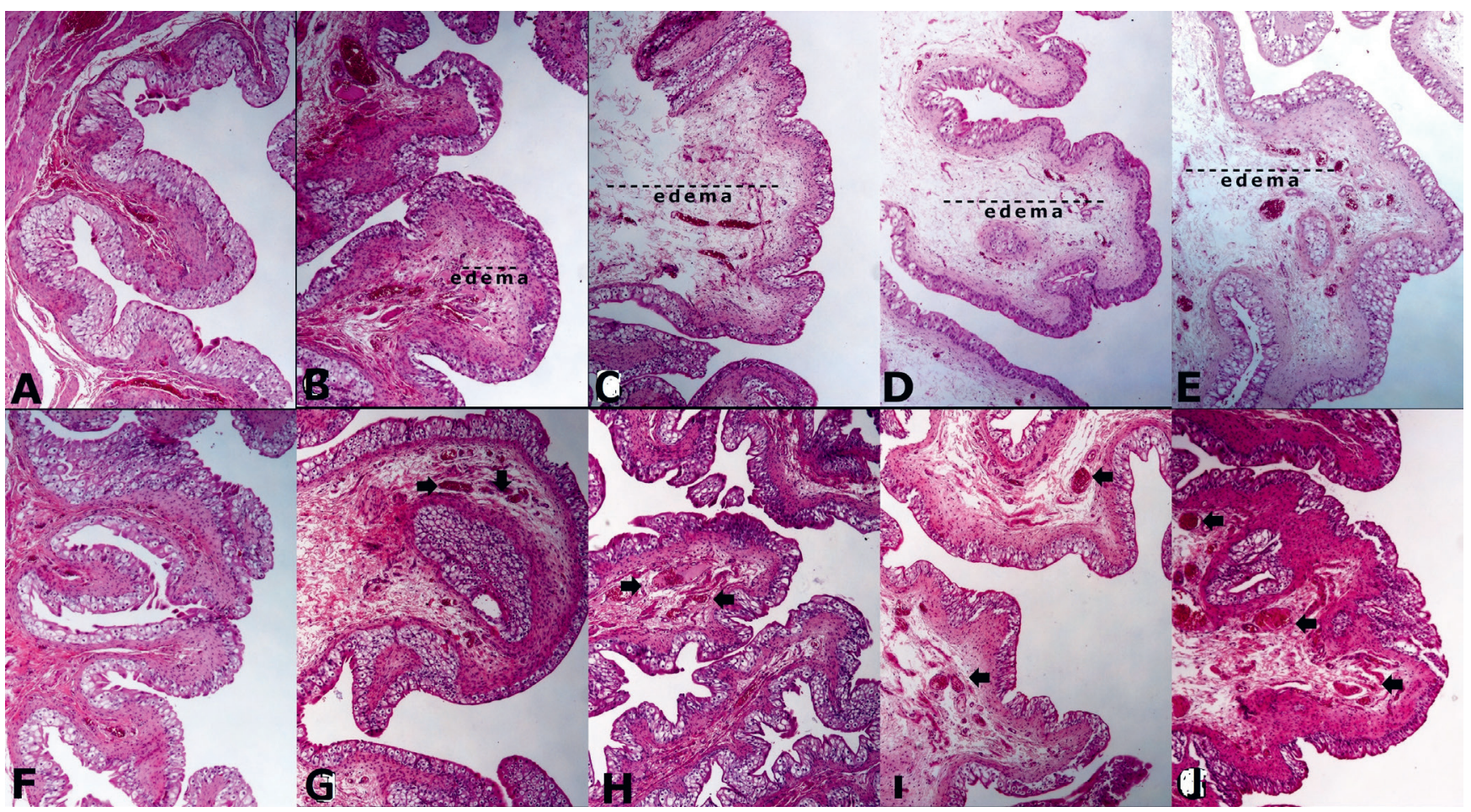

Fig. 1. Histopathological view of group in terms of edema and congestion. A. Control group with no edema (group 1). B. Mild-moderate edema (group 3). C, D, E. Severe edema (groups 2, 4 and 5). F. Control group no congestion (group 1). G. Mild congestion (group 5). H, I, J. Moderate congestion (groups 2, 3 and 4) (arrows). Hematoxylin \& eosin (H\&E) staining, magnification $\times 40$

However, compared to the control group (comparison of groups 1 and 3), edema was still significantly higher $(\mathrm{p}<0.01)$ (Table 1, Fig. 1). There was a statistically significant increase in congestion in groups 3,4 and 5 compared to the control group $(\mathrm{p}<0.001)$. When compared to the other groups with SCI, in group 5, the congestion was less pronounced, but there was not statistically significant difference ( $\mathrm{p}=0.02)$ (Table 1, Fig. 1).

As a result, combined treatment were found to be slightly more effective than MP alone in reducing edema while no significant effect was observed in preventing early inflammation in the early (early $24 \mathrm{~h}$ ) period after SCI. Congestion was slightly less observed in the DNT+MP group (Fig. 2C).

\section{Biochemical findings}

\section{Biochemical findings in blood}

The blood MDA level after SCI was significantly increased compared to the control group ( $\mathrm{p}<0.01)$. All 3 drugs showed similar efficacy for lowering the elevated MDA levels after SCI and made blood MDA levels similar to the control group (Table 1, Fig. 3A).

When the blood GSH level after SCI was compared with the control group, it fell significantly in all groups $(\mathrm{p}<0.01$ and $\mathrm{p}<0.05)$. Dantrolene and MP alone did not have a significant effect on increasing the lowered 

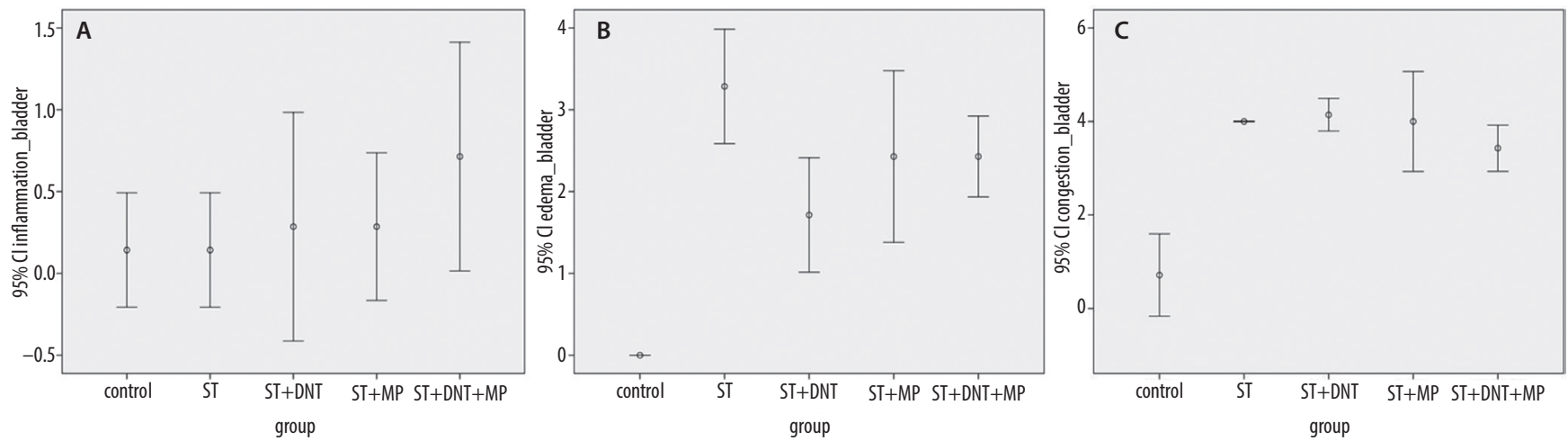

Fig. 2. Comparison of inflammation, edema and congestion in experimental groups. A. There was no difference among all the groups in terms of inflammation. B. Compared to the control group, there was a significant increase in edema in all other groups $(p=0.000)$. There was no difference between groups 4 and 5. Edema was less observed in the group in which DNT was administered alone. C. There was a significant increase in congestion in the $\mathrm{SCl}$ and drug-administered groups $(p<0.001)$, congestion was less observed in the DNT+MP group

ST - spinal trauma; DNT - dantrolene; MP - methylprednisolone.
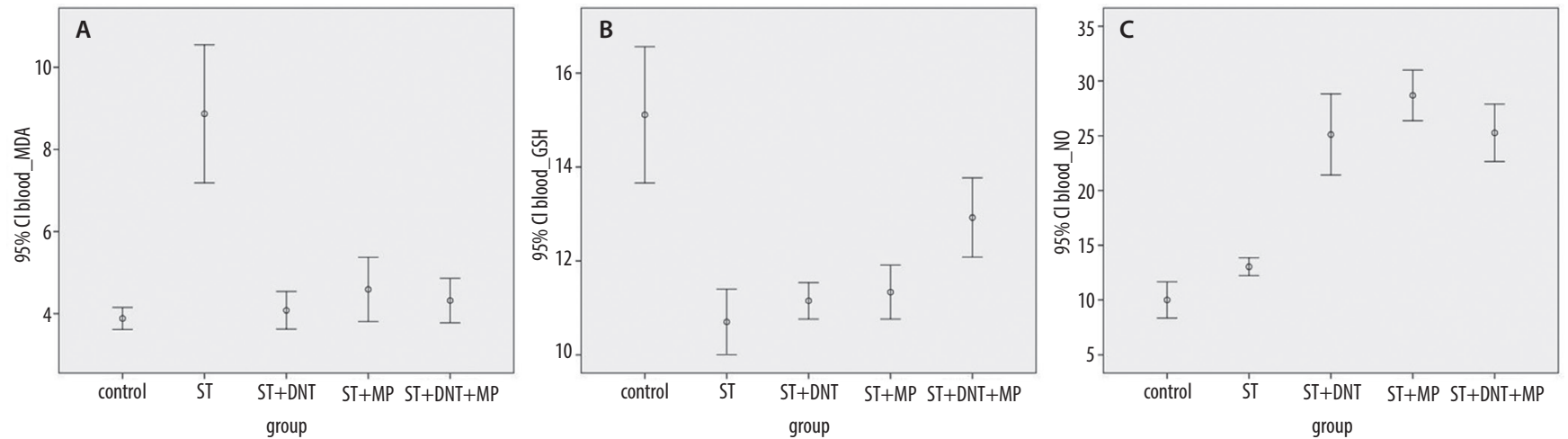

Fig. 3. Comparison of blood MDA, GSH and NO levels in experimental groups. A. Blood MDA levels fell in all 3 drug-treated groups, reaching the same level as in the control group. B. Blood GSH values were decreased after SCI, and neither DNT nor MP did not have a statistically significant effect; GSH levels were highest in DNT+MP group. C. Blood NO values were significantly increased in all 3 drug-administered groups, but the increase was most observed in the MP-administered group

ST - spinal trauma; DNT - dantrolene; MP - methylprednisolone; GSH - glutathione.

blood GSH level ( $\mathrm{p}=0.22$ and $\mathrm{p}=0.13$, respectively). When the groups treated with DNT and MP alone were compared to the control group, the blood GSH level was significantly lower $(\mathrm{p}<0.01)$. After SCI, the increase in blood GSH levels was observed most prominently in the DNT+MP group, but nevertheless it could not reach the blood GSH level in the control group $(\mathrm{p}<0.05)$ (Table 1, Fig. 3B).

After SCI, the blood NO level was significantly higher in comparison to the control group ( $\mathrm{p}<0.005)$, and all 3 groups which received drug therapy had significantly raised levels of $\mathrm{NO}$ in comparison to the control group as well as the SCI group. When compared to the control group, blood NO levels were highest in the group which received only MP among the 3 groups which received drugs $(\mathrm{p}<0.01)$ (Table 1, Fig. 3C).

As a result, use of DNT and MP alone or in combination was successful in lowering blood MDA levels and the most successful group for increasing GSH level after $\mathrm{SCI}$ was the DNT+MP group. Blood NO levels were highest in the group which received only MP.

\section{Biochemical findings in bladder tissue}

When MDA levels in the bladder were examined, the level of MDA after SCI was significantly increased in the bladder tissue. Similar efficacy was observed in all 3 treated groups in decreasing MDA levels which were increased after SCI. Malondialdehyde levels in the bladder tissue decreased to the level of the control group in each of the 3 drug-administered groups (Table 1, Fig. 4A). For the level of GSH in the bladder tissue, no significant change was observed between the experiment groups (Table 1, Fig. 4B). The NO level in bladder tissue was significantly decreased in the DNT and DNT+MP groups and fell below the values of the control group $(\mathrm{p}<0.05)$ (Table 1, Fig. 4C).

\section{Discussion}

The aim of this study was to compare the protective efficacy of DNT and MP alone and in combination in preventing early sequelae associated with oxidative stress 

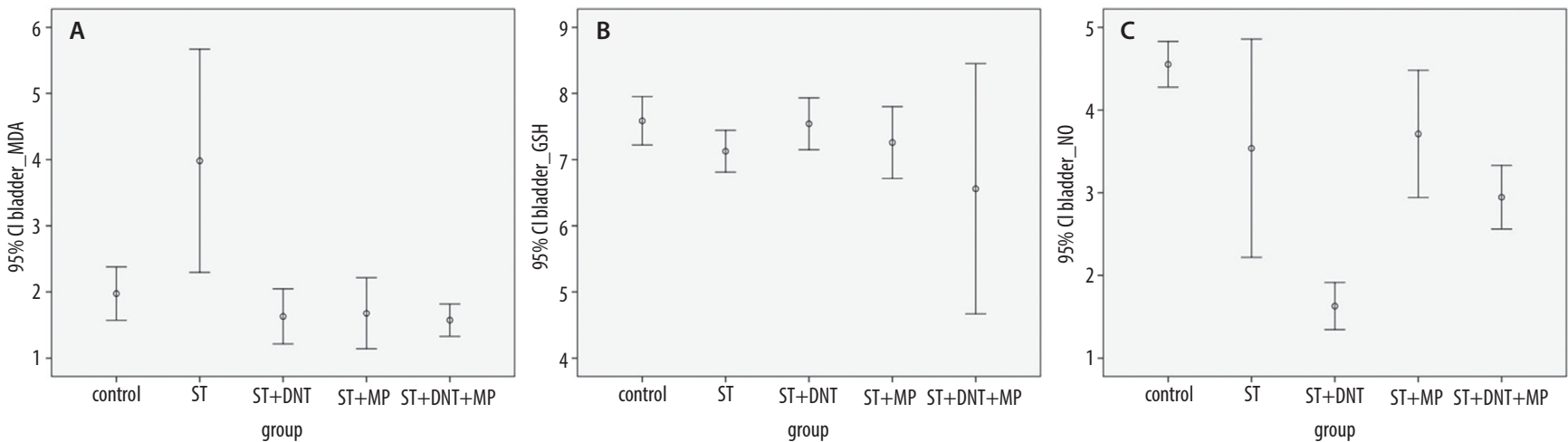

Fig. 4. Comparison of bladder tissue MDA, GSH and NO levels in experimental groups. A. After SCl, bladder MDA levels were significantly increased, and tissue MDA levels decreased in each of the 3 drug-administered groups when compared to the control group. B. There was no significant change in bladder tissue GSH levels among the experimental groups. C. In the group receiving DNT and DNT+MP, bladder tissue NO levels were significantly decreased after $\mathrm{SCl}$ and fell below the values of the control group $(p<0.05)$

ST - spinal trauma; DNT - dantrolene; MP - methylprednisolone; GSH - glutathione.

secondary to traumatic SCI. For this reason, their effects in the first $24 \mathrm{~h}$ after trauma were investigated.

Spinal cord injury remains an important clinical problem that can still lead to persistent neurological deficits and secondary complications. ${ }^{5}$ In patients with SCI, multiple organ dysfunction and failure may develop. ${ }^{2}$ These disorders include neurogenic pain and depression in the nervous system, orthostatic hypotension and autonomic dysreflexia in the cardiovascular system, spleen atrophy and leukopenia, pulmonary edema in the lungs, muscle spasticity and atrophy in the skeletal system, osteoporosis in the bone, neurogenic bowel dysfunction in the gastrointestinal system, renal damage in the urinary system, neurogenic bladder and urinary tract infection, and sexual dysfunction. ${ }^{2}$ In addition, SCI can trigger a systemic inflammatory response syndrome, which can be life-threatening by affecting the distal organs. ${ }^{2}$ Significant structural, molecular and physiological changes have been reported to develop in SCI animal model studies. In these animal models, hemorrhage due to cellular inflammatory response, rupture of the bladder uroepithelium and inflammation were observed. ${ }^{16-20}$ Primary injury in SCI is unavoidable. However, measures against the development of secondary injury can be taken while the treatments are applied. Research and treatments performed for this reason are aimed to prevent secondary injury. ${ }^{5}$ Reduction of oxidative stress leading to membrane and cellular damage can provide a potential treatment to prevent secondary injury. ${ }^{21}$ A number of studies have been conducted for the treatment of secondary injuries and some therapeutic agents have been used in SCI. Some of the therapeutic agents used for this purpose are DNT and MP. However, there is still no effective treatment to prevent the effects of secondary injury. ${ }^{4}$

After SCI, changes in antioxidant enzyme activities such as superoxide dismutase (SOD), catalase (CAT) and glutathione peroxidase (GPx) can be observed. Decreased SOD and GPx activities in rats after SCI have been reported in the literature. ${ }^{22,23}$ In contrast, Nishibe et al., reported no change in total SOD activity in SCI-induced dogs, but a significant reduction in CAT activity was reported. ${ }^{24}$ In an experimental study by Cavus et al., in which SCI was induced in rats, it was found that SOD levels were significantly higher in the trauma group and no significant difference was found in GPx and CAT levels between the groups. ${ }^{10}$ In our experimental study, GSH, MDA and NO levels were investigated through a biochemical analysis of blood and bladder tissue.

After SCI, systemic inflammation may be triggered and inflammation may develop in distal organs. ${ }^{2}$ Irregularities in the neuroendocrine system and changes in neuroimmune regulation are the determining factors in the onset and progression of systemic inflammation after SCI. ${ }^{2}$ Spinal cord injury activates the hypothalamic-pituitary-adrenal axis, leading to an increase in the macrophage migration inhibitory factor produced by the pituitary gland. Macrophage migration inhibitory factor is thought to play an important role in the progression of systemic inflammation. ${ }^{2}$ Acute treatment after SCI may contribute to healing by suppressing neuroinflammation. ${ }^{25,26}$ Studies of SCI in animal models have reported that hemorrhage and inflammatory changes occur in the post-SCI period. Torres et al. found in their study about bladder morphology in SCI rabbits that there was a significant increase in hemorrhage and inflammation in the bladder after $32 \mathrm{~h}$ of SCI, and inflammatory infiltration in the bladder was reported to be significantly less pronounced in the DNT group on day $8 .^{16}$ Anti-inflammatory treatment may be beneficial in the treatment of neurogenic bladder by inhibiting the inflammatory response. ${ }^{2,27}$ Studies have reported that antioxidants such as DNT after SCI contribute to healing of mesenteric lesions by reducing hemorrhage and immunocyte infiltration in the bladder. $2,16,20$ In our current study, unlike the literature, there was no difference between the experimental groups in terms of inflammation in the bladder. This may be due to the inadequate development of inflammation in the first $24 \mathrm{~h}$. If more than $24 \mathrm{~h}$ of changes had been investigated, perhaps different results could be obtained in terms of edema and inflammation. When compared to the control group, 
it was observed that all groups had increased bladder tissue edema. Edema in the DNT group was less pronounced than in other groups which received other drugs. When compared to the control group, it was observed that all groups had increased bladder congestion. Among the drugadministered groups, the DNT+MP combination group was found to be the group with the least congestion.

In traumatic SCI, lipid peroxidation is one of the important trigger components of neuronal degeneration. The increase in lipid peroxidation may be due to an insufficiency of enzymatic and non-enzymatic defense mechanisms. For this reason, the prevention of lipid peroxidation may be important for neurological recovery. Malondialdehyde results from the effect of reactive oxygen radicals on membrane lipids. It is one of the most important indicators of lipid peroxidation and its blood and tissue levels increases after oxidative stress. ${ }^{4,20,28}$ In studies conducted, it has been reported that MDA levels in the blood and tissues are increased in animal models with traumatic SCI. In the study of Aslan et al., it was reported that blood MDA levels were significantly increased, and after using DNT blood MDA levels significantly decreased in animal models of SCI. In the same study, it was reported that the MDA level in cerebrospinal fluid significantly increased after DNT treatment but there was no significant change of MDA level in spinal cord tissue. ${ }^{5}$ In another study of animal models of SCI, increased MDA levels were reported to be significantly reduced in MPtreated groups. ${ }^{28}$ In our study, an increase in MDA levels was also detected in the bladder tissue along with the increase in the levels of the corresponding MDA levels in blood. Reducing the level of MDA, an oxidative stress indicator, may be effective in reducing oxidative stress-related damage. Single or combined use of DNT, which has anti-lipid peroxidative and neuroprotective properties, or MP, which is a glucocorticoid agent with anti-inflammatory properties, reduced MDA levels both in blood and bladder tissue. These findings are evidence supporting the clinical utility of DNT and MP in preventing oxidative damage in post-traumatic SCI.

Glutathione, an important cellular antioxidant, is a thiolcontaining tripeptide. It has important biological functions in the defense against the potential damage of oxidative stress. Glutathione protects the cells from possible damage by reacting with free radicals. ${ }^{5,28,29}$ Decreased levels of GSH during increased oxidative stress have been reported. A significant increase in GSH levels was observed after MP treatment in experimental animal models of SCI designed by Ates et al. ${ }^{28}$ In another study, GSH levels decreased significantly after DNT therapy in animal models of SCI. However, spinal fluid and spinal cord tissue GSH levels were not reported to increase significantly in response to DNT therapy. ${ }^{5}$ Cevik et al. investigated the effect of quercetin on rat bladder after SCI and found a significant decrease in GSH levels after SCI in the bladder tissue. ${ }^{20}$ In our present work, there was a significant decrease in blood GSH levels after SCI, but there was no significant change in GSH levels in bladder tissue after
SCI. The decrease in blood GSH levels in SCI animal models is consistent with the results reported in the literature. No significant change in GSH levels in the bladder tissue of the experimental groups was a similar result as in the literature in which no change in GSH levels was reported in cerebrospinal fluid and the spinal cord. However, the decrease in GSH level in the bladder tissue was not found to be significant compared to the literature. These results suggest that the evaluation of post-SCI levels of GSH in blood is more valuable than evaluating tissue samples. In our study, it was found that DNT and MP had no effect on increasing blood GSH levels when used as single therapy, but showed a significant effect when combined, increasing blood GSH levels. Therefore, combined treatment of DNT and MP at different doses should be investigated as a new therapeutic alternative to increase the level of GSH, which is an important antioxidant.

Nitric oxide is an inorganic free radical gas molecule produced from L-arginine under the influence of NO synthase isoenzymes (iNOS, nNOS and eNOS). ${ }^{28,30}$ Nitric oxide is a molecule with both antioxidant and pro-oxidant properties. It is a chain-breaking antioxidant in free radical-mediated lipid peroxidation. ${ }^{4,31,32}$ It is involved in some physiological processes, such as regulation of blood vessel walls and neurotransmission, when within physiological limits. However, in situations such as oxidative stress, excessive elevations in NO levels may be detrimental to tissues. ${ }^{28,33}$ In non-pathological situations, the NO concentration is in the nanomolar range, but in oxidative damage situations - in the micromolar range. ${ }^{31,32}$ In SCI animal models, there was a significant increase in NO levels in the spinal cord after trauma, and there was a significant decrease in tissue NO levels in the MP group. ${ }^{28}$ In another study, NO levels were assessed in experimental rabbits with SCI which were given DNT, and there was no difference between the experimental groups in terms of blood and cerebrospinal fluid NO levels. ${ }^{5}$ Similar to studies that reported an increase in NO levels after oxidative stress, we also observed a significant increase in blood NO levels in all rabbits after SCI compared to the control group. In experimental groups receiving DNT and DNT+MP, NO levels in bladder tissue also decreased significantly. In our study, DNT and MP were observed to cause a decrease in NO levels in the bladder tissue. This result supports studies that reported a decrease in NO levels in spinal cord and bladder tissue in response to anti-inflammatory treatment in SCI animal models.

In summary, there was no significant increase of inflammation in the bladder tissue at the early stage after traumatic SCI, but there was a significant increase in edema and congestion. There was a significant increase in MDA levels in both blood and bladder tissue. On the other hand, for GSH levels, there was a significant decline in blood, but there was no significant change in bladder tissue. A significant increase was observed in NO levels after traumatic SCI. For reducing edema, use of DNT only was significantly effective, and for reducing congestion, combined 
use of DNT and MP was found to be effective. The blood NO level was highest in the MP group. In bladder tissue, the DNT and DNT+MP groups showed a significant decrease in NO level. While single or combined use of DNT and MP was successful in reducing blood MDA levels and no superiority was observed between them, it was observed that DNT+MP was more effective in increasing the level of GSH after oxidative stress.

Such experimental studies are very important in the development of treatment strategies aimed at improving human health after post-traumatic SCI. Potential therapeutic benefits of single or combined use of DNT and MP, along with other treatment approaches, may be seen in reducing the effects of oxidative stress and secondary damage in the bladder following traumatic SCI. The results obtained in our experimental study suggest that combined use of DNT and MP after SCI can be more effective and beneficial in preventing the formation of damage due to oxidative stress in the bladder, providing additional protection. For this reason, the combined use of DNT and MP can be considered as a promising therapeutic strategy. Human clinical trials with an extensive series of treatment strategies in different doses and combined use of DNT and MP are needed in preventing post-SCI damage in the bladder after SCI. In the future, with welldesigned experimental studies, it will be a more realistic approach to apply the results of DNT and MP in different doses and combined administration in clinical practice.

\section{References}

1. Lasfargues JE, Custis D, Morrone F, et al. A model for estimating spinal cord injury prevalence in the United States. Paraplegia. 1995;33(2): 62-68.

2. Sun X, Jones ZB, Chen XM, Zhou L, So KF, Ren Y. Multiple organ dysfunction and systemic inflammation after spinal cord injury: A complex relationship. J Neuroinflammation. 2016;3(1):260.

3. Wu J, Yang H, Qiu Z, Zhang Q, Ding T, Geng D. Effect of combined treatment with methylprednisolone and Nogo-A monoclonal antibody after rat spinal cord injury. J Int Med Res. 2010;38(2):570-582.

4. Anwar MA, Al Shehabi TS, Eid AH. Inflammogenesis of secondary spinal cord injury. Front Cell Neurosci. 2016;10:98.

5. Aslan A, Cemek M, Buyukokuroglu ME, et al. Dantrolene can reduce secondary damage after spinal cord injury. Eur Spine J. 2009;18(10): 1442-1451.

6. Tator $\mathrm{CH}$. Review of experimental spinal cord injury with emphasis on the local and systemic circulatory effects. Neurochirurgie. 1991; 37(5):291-302.

7. Eaton MJ. Cell and molecular approaches to the attenuation of pain after spinal cord injury. J Neurotrauma. 2006;23(3-4):549-559.

8. Tator $\mathrm{CH}$, Fehlings MG. Review of the secondary injury theory of acute spinal cord trauma with emphasis on vascular mechanisms. J Neurosurg. 1991;75(1):15-26.

9. Liu D, Li L, Augustus L. Prostaglandin release by spinal cord injury mediates production of hydroxyl radical, malondialdehyde and cell death: A site of the neuroprotective action of methylprednisolone. J Neurochem. 2001;77(4):1036-1047.

10. Cavus G, Altas M, Aras M, et al. Effects of montelukast and methylprednisolone on experimental spinal cord injury in rats. Eur Rev Med Pharmacol Sci. 2014;18(12):1770-1777.
11. Rosado IR, Lavor MSL, Alves EGL, et al. Effects of methylprednisolone, dantrolene, and their combination on experimental spinal cord injury. Int J Clin Exp Pathol. 2014;7(8):4617-4626.

12. Ohkawa $\mathrm{H}$, Ohishi $\mathrm{N}$, Yagi K. Assay for lipid peroxides in animal tissues by Thiobarbituric acid reaction. Anal Biochem. 1979;95(2):351-358.

13. Beutler E, Dubon OB, Kelly M. Improved method for the determination of blood glutathione. J Lab Clin Med. 1963;61:882-888.

14. Somogyi M. A method for the preparation of blood filtrates for the determination of sugar. J Biol Chem. 1930;86:55.

15. Miranda KM, Espey MG, Wink DA. A rapid, simple spectrophotometric method for simultaneous detection of nitrate and nitrite. Nitric Oxide. 2001;5(1):62-71.

16. Torres B, Serakides R, Caldeira F, Gomes M, Melo E. The ameliorating effect of dantrolene on the morphology of urinary bladder in spinal cord injured rats. Pathol Res Pract. 2011;207(12):775-779.

17. Apodaca G, Kiss S, Ruiz W, Meyers S, Zeidel M, Birder L. Disruption of bladder epithelium barrier function after spinal cord injury. Am J Physiol Renal Physiol. 2003;284(5):966-976.

18. Herrera JJ, Haywood-Watson RJ $2^{\text {nd }}$, Grill RJ. Acute and chronic deficits in the urinary bladder after spinal contusion injury in the adult rat. J Neurotrauma. 2010;27(2):423-431.

19. Leung PY, Johnson CS, Wrathall JR. Comparison of the effects of complete and incomplete spinal cord injury on lower urinary tract function as evaluated in unanesthetized rats. Exp Neurol. 2007;208(1):80-91.

20. Cevik O, Ersahin M, Sener TE, et al. Beneficial effects of quercetin on rat urinary bladder after spinal cord injury. J Surg Res. 2013;183(2): 695-703.

21. Chen X, Cui J, Zhai X, et al. Inhalation of hydrogen of different concentrations ameliorates spinal cord injury in mice by protecting spinal cord neurons from apoptosis, oxidative injury and mitochondrial structure damages. Cell Physiol Biochem. 2018;47(1):176-190.

22. Kanter M, Coskun O, Kalayci M, Buyukbas S, Cagavi F. Neuroprotective effects of Nigellasativa on experimental spinal cord injury in rats. Hum Exp Toxicol. 2006;25(3):127-133.

23. Erol FS, Kaplan M, Tiftikci M, et al. Comparison of the effects of octreotide and melatonin in preventing nerve injury in rats with experimental spinal cord injury. J Clin Neurosci. 2008;15(7):784-790.

24. Nishibe M. Experimental studies on the mechanism of spinal cord ischemia the state of free radical scavengers [in Japanese]. Hokkaido Igaku Zasshi. 1989;64(3):301-308.

25. Patel SP, Cox DH, Gollihue JL, et al. Pioglitazone treatment following spinal cord injury maintains acute mitochondrial integrity and increases chronic tissues paring and functional recovery. Exp Neurol. 2017;293:74-82.

26. Cabrera Aldana EE, Ruelas F, Aranda C, et al. Methylprednisolone administration following spinal cord injury reduces aquaporin 4 expression and exacerbates edema. Mediators Inflamm. 2017;2017: 4792932.

27. Shunmugavel A, Khan M, Hughes Jr FM, Purves JT, Singh A, Singh I. S-Nitroso glutathione protects the spinal bladder: Novel therapeutic approach to post-spinal cord injury bladder remodeling. Neurourol Urodyn. 2015;34(6):519-526.

28. Ates $\mathrm{O}, \mathrm{Cayli} \mathrm{S}$, Altinoz $\mathrm{E}$, et al. Effects of resveratrol and methylprednisolone on biochemical, neurobehavioral and histopathological recovery after experimental spinal cord injury. Acta Pharmacol Sin. 2006;27(10):1317-1325.

29. Meister A, Anderson ME. Glutathione. Annu Rev Biochem. 1983;52: 711-760.

30. Moncada S, Higgs A. The L-arginine-nitric oxide pathway. N Engl J Med. 1993;329(27):2002-2012.

31. Taysi S, Koc M, Büyükokuroğlu ME, Altinkaynak K, Sahin YN. Melatonin reduces lipid peroxidation and nitric oxide during irradiation-induced oxidative injury in the rat liver. J Pineal Res. 2003;34(3):173-177.

32. Joshi MS, Ponthier JL, Lancaster JR Jr. Cellular antioxidant and prooxidant actions of nitric oxide. Free Radic Biol Med. 1999;27(11-12): 1357-1366.

33. Von Euler M, Akesson E, Samuelsson EB, Seiger A, Sundstrom E. Motor performance score: A new algorithm for accurate behavioral testing of spinal cord injury in rats. Exp Neurol. 1996;137(2):242-254. 